\title{
Existence and Uniqueness of Solutions to Neutral Stochastic Functional Differential Equations with Poisson Jumps
}

\author{
Jianguo Tan, ${ }^{1}$ Hongli Wang, ${ }^{2}$ and Yongfeng Guo ${ }^{1}$ \\ ${ }^{1}$ Department of Mathematics, Tianjin Polytechnic University, Tianjin 300387, China \\ ${ }^{2}$ Department of Mechanics, Tianjin University, Tianjin 300072, China
}

Correspondence should be addressed to Jianguo Tan, tanjg@tju.edu.cn

Received 9 March 2012; Revised 12 May 2012; Accepted 15 May 2012

Academic Editor: Márcia Federson

Copyright (C) 2012 Jianguo Tan et al. This is an open access article distributed under the Creative Commons Attribution License, which permits unrestricted use, distribution, and reproduction in any medium, provided the original work is properly cited.

A class of neutral stochastic functional differential equations with Poisson jumps (NSFDEwPJs), $\mathrm{d}\left[x(t)-G\left(x_{t}\right)\right]=f\left(x_{t}, t\right) \mathrm{d} t+g\left(x_{t}, t\right) \mathrm{d} W(t)+h\left(x_{t}, t\right) \mathrm{d} N(t), t \in\left[t_{0}, T\right]$, with initial value $x_{t_{0}}=$ $\xi=\{\xi(\theta):-\tau \leq \theta \leq 0\}$, is investigated. First, we consider the existence and uniqueness of solutions to NSFDEwPJs under the uniform Lipschitz condition, the linear growth condition, and the contractive mapping. Then, the uniform Lipschitz condition is replaced by the local Lipschitz condition, and the existence and uniqueness theorem for NSFDEwPJs is also derived.

\section{Introduction}

Neutral stochastic functional differential equations (NSFDEs) have recently been studied intensively, for instance, Mao [1] and Kolmanovskii [2], Mao et al. [3-6], Luo et al. [7], Zhou and $\mathrm{Hu}$ [8], and Luo [9]. Poisson jumps are becoming increasingly used to model real-world phenomena in different fields such as economics, finance, biology, and physics. There is an extensive literature concerned with Poisson jumps, for example, Wang et al. $[10,11]$, Ronghua et al. [12, 13], Luo [14], and Tan and Wang [15]. Therefore, it is natural and necessary to incorporate jumps in the neutral stochastic functional differential equations. However, the study of NSFDEwPJs is limited by far. Liu et al. [16] studied the stability of NSFDEwPJs by using fixed point theory, Luo and Taniguchi [17] proved the existence and uniqueness of non-Lipschitz stochastic neutral delay evolution equations driven by Poisson jumps. However, no theory for the existence and uniqueness of solutions to NSFDEwPJs has 
been established yet. Therefore, in this paper, we first prove the existence and uniqueness of solutions to NSFDEwPJs.

The outline of the paper is as follows. In Section 2 we will introduce some necessary notations and assumptions. In Section 3, we will present several useful lemmas, and then we prove the existence and uniqueness of NSFDEwPJs under the uniform Lipschitz condition, the linear growth condition, and the contractive mapping. Furthermore, the uniform Lipschitz condition is replaced by the local Lipschitz condition, and the existence and uniqueness theorem is also derived.

\section{Preliminaries}

Let $(\Omega, \mathcal{F}, P)$ be a complete probability space with a filtration $\left\{\mathcal{F}_{t}\right\}_{t \geq 0}$, which satisfies the usual conditions, that is, the filtration is continuous on the right and $\mathcal{F}_{0}$ contains all $P$ null sets. Moreover, $C\left([a, b] ; R^{n}\right)$ denotes the family of functions $\varphi$ from $[a, b]$ to $R^{n}$ that are right-continuous and have limits on the left, $C\left([a, b] ; R^{n}\right)$ is equipped with the norm $\|\psi\|=\sup _{a<t<b}|\psi(t)|$, where $|\cdot|$ is the Euclidean norm in $R^{n}$, that is, $|x|=\sqrt{x^{T} x}\left(x \in R^{n}\right)$. Let $\mathcal{L}^{p}\left([a, b] ; R^{n}\right)$ denote the family of all $R^{n}$-valued $\mathcal{F}_{t}$-adapted process $f(t), t \in[a, b]$, such that $\int_{a}^{b}|f(t)|^{p} \mathrm{~d} t<\infty$. We denote by $\mathcal{M}^{2}\left([-\tau, 0] ; R^{n}\right)$ the family of the processes $\xi(t)$ in $\mathcal{L}^{2}\left([-\tau, 0] ; R^{n}\right)$ such that $E \int_{-\tau}^{0}|\xi(t)|^{2} \mathrm{~d} t<\infty . a \vee b$ means the maximum of $a$ and $b$, and $a \wedge b$ means the minimum of $a$ and $b$.

In this paper, we consider the $n$-dimensional neutral stochastic functional differential equations with Poisson jumps

$$
\mathrm{d}\left[x(t)-G\left(x_{t}\right)\right]=f\left(x_{t}, t\right) \mathrm{d} t+g\left(x_{t}, t\right) \mathrm{d} W(t)+h\left(x_{t}, t\right) \mathrm{d} N(t), \quad t \in\left[t_{0}, T\right],
$$

where $x_{t}=\{x(t+\theta):-\tau \leq \theta \leq 0\}$ can be regarded as a $C\left([-\tau, 0] ; R^{n}\right)$-valued stochastic process, $W(t)$ is an $m$-dimensional standard Wiener process that is $\mathbb{f}_{t}$-adapted, and $N(t)$ is a scalar Poisson process with intensity $\lambda$. Assume that $W(t)$ and $N(t)$ are independent of $\mathcal{F}_{t_{0}}$. Moreover, the functions $G: C\left([-\tau, 0] ; R^{n}\right) \rightarrow R^{n}, f: C\left([-\tau, 0] ; R^{n}\right) \times\left[t_{0}, T\right] \rightarrow R^{n}$, $g: C\left([-\tau, 0] ; R^{n}\right) \times\left[t_{0}, T\right] \rightarrow R^{n \times m}, h: C\left([-\tau, 0] ; R^{n}\right) \times\left[t_{0}, T\right] \rightarrow R^{n}$.

The stochastic integral is defined in the Itô sense, and the integral version of (2.1) is frequently expressed as

$$
x(t)-G\left(x_{t}\right)=x\left(t_{0}\right)-G\left(x_{t_{0}}\right)+\int_{t_{0}}^{t} f\left(x_{s}, s\right) \mathrm{d} s+\int_{t_{0}}^{t} g\left(x_{s}, s\right) \mathrm{d} W(s)+\int_{t_{0}}^{t} h\left(x_{s}, s\right) \mathrm{d} N(s) .
$$

The initial value is followed:

$$
\begin{aligned}
x_{t_{0}}= & \xi=\{\xi(\theta):-\tau \leq \theta \leq 0\} \text { is } \mathcal{F}_{t_{0}}-\text { measurable } C\left([-\tau, 0] ; R^{n}\right) \\
& \text { - valued random variable such that } \xi \in \mathcal{M}^{2}\left([-\tau, 0] ; R^{n}\right)
\end{aligned}
$$




\section{The Existence and Uniqueness Theorem}

Definition 3.1. An $R^{n}$-valued stochastic process $x(t)$ on $t_{0}-\tau \leq t \leq T$ is called a solution to (2.1) with initial data (2.3) if it has the following properties:

(i) $x(t)$ is continuous and $\left\{x_{t}\right\}_{t_{0} \leq t \leq T}$ is $\mathcal{F}_{t}$-adapted;

(ii) $f\left(x_{t}, t\right) \in \mathcal{L}^{1}\left(\left[t_{0}, T\right] ; R^{n}\right)$ and $g\left(x_{t}, t\right) \in \mathcal{L}^{2}\left(\left[t_{0}, T\right] ; R^{n \times m}\right)$;

(iii) $x_{t_{0}}=\xi$ and (2.2) hold for each $t_{0} \leq t \leq T$. it, that is,

A solution $x(t)$ is said to be unique if any other solution $\bar{x}(t)$ is indistinguishable from

$$
P\left\{x(t)=\bar{x}(t) \forall t_{0}-\tau \leq t \leq T\right\}=1
$$

Let us now begin to establish the theory of the existence and uniqueness of the solution to (2.1) with initial data (2.3).

Theorem 3.2. Assume that there exist two positive constants $\bar{K}$ and $K$ such that

(H1) (the Lipschitz condition) For all $\phi, \varphi \in C\left([-\tau, 0] ; R^{n}\right)$ and $t \in\left[t_{0}, T\right]$,

$$
|f(\phi, t)-f(\varphi, t)|^{2} \vee|g(\phi, t)-g(\varphi, t)|^{2} \vee|h(\phi, t)-h(\varphi, t)|^{2} \leq \bar{K}\|\phi-\varphi\|^{2} .
$$

(H2) (the linear growth condition) for all $(\phi, t) \in C\left([-\tau, 0] ; R^{n}\right) \times\left[t_{0}, T\right]$,

$$
|f(\phi, t)|^{2} \vee|g(\phi, t)|^{2} \vee|h(\phi, t)|^{2} \leq K\left(1+\|\phi\|^{2}\right) .
$$

(H3) (the constractive mapping) there is a positive constant $\mathcal{\kappa} \in(0,1)$ such that, for all $\phi, \varphi \in$ $C\left([-\tau, 0] ; R^{n}\right)$,

$$
|G(\phi)-G(\varphi)| \leq \kappa\|\phi-\varphi\|
$$

Then, there exists a unique solution $x(t)$ to (2.1) with initial data (2.3). Moreover, the solution belongs to $\mathcal{M}^{2}\left(\left[t_{0}-\tau, T\right] ; R^{n}\right)$.

To prove Theorem 3.2, we give the following useful Lemmas 3.3 and 3.4 first.

Lemma 3.3. If $p \geq 2, g \in \mathcal{M}^{2}\left(\left[t_{0}, T\right] ; R^{n \times m}\right)$ such that

$$
E \int_{t_{0}}^{t}|g(s)|^{p} d s<\infty
$$

then $E\left|\int_{t_{0}}^{T} g(s) d W(s)\right|^{p} \leq(p(p-1) / 2)^{p / 2} T^{(p-2) / 2} E \int_{t_{0}}^{T}|g(s)|^{p} d s$.

In particular, for $p=2$, there is equality.

The proof of Lemma 3.3 can be found in [6]. 
Lemma 3.4. For any $a, b>0$ and $0<\alpha<1$ one has

$$
(a+b)^{2} \leq \frac{a^{2}}{\alpha}+\frac{b^{2}}{1-\alpha}
$$

The proof of Lemma 3.4 can also be found in [6].

To prove the uniqueness of the solution for (2.1), we will establish Lemma 3.5.

Lemma 3.5. Let (3.3) and (3.4) hold. If $x(t)$ is a solution to (2.1) with initial data (2.3), then

$$
\begin{aligned}
E\left(\sup _{t_{0}-\tau \leq t \leq T}|x(t)|^{2}\right) \leq & \left(1+\frac{5+\kappa \sqrt{\kappa}}{(1-\kappa)(1-\sqrt{\kappa})} E\|\xi\|^{2}\right) \\
& \times \exp \left[\frac{4 K\left(T-t_{0}\right)\left(T-t_{0}+4 K+8 \lambda+2 \lambda^{2}\left(T-t_{0}\right)\right)}{(1-\kappa)(1-\sqrt{\kappa})}\right],
\end{aligned}
$$

where $\mathcal{\kappa}$ is the contraction constant in (3.4). In particular, $x(t)$ belongs to $\mathcal{M}^{2}\left(\left[t_{0}-\tau, T\right] ; R^{n}\right)$.

Proof. For every integer $n \geq 1$, define the stopping time

$$
\tau_{n}=T \wedge \inf \left\{t \in\left[t_{0}, T\right]:\left\|x_{t}\right\| \geq n\right\}
$$

Clearly, $\tau_{n} \uparrow T$ a.s. Set $x^{n}(t)=x\left(t \wedge \tau_{n}\right)$ for $t \in\left[t_{0}, T\right]$. Then, for $t_{0} \leq t \leq T$

$$
\begin{aligned}
x^{n}(t)= & G\left(x_{t}^{n}\right)-G(\xi)+\xi(0)+\int_{t_{0}}^{t} f\left(x_{s}^{n}, s\right) I_{\left[t_{0}, \tau_{n}\right]}(s) \mathrm{d} s \\
& +\int_{t_{0}}^{t} g\left(x_{s}^{n}, s\right) I_{\left[t_{0}, \tau_{n}\right]}(s) \mathrm{d} W(s)+\int_{t_{0}}^{t} h\left(x_{s}^{n}, s\right) I_{\left[t_{0}, \tau_{n}\right]}(s) \mathrm{d} N(s),
\end{aligned}
$$

where $I_{A}$ is the indicator function of a set $A$, that is,

$$
I_{A}(x)= \begin{cases}1, & x \in A, \\ 0, & x \notin A .\end{cases}
$$

Set

$$
\begin{aligned}
J^{n}(t)= & \xi(0)+\int_{t_{0}}^{t} f\left(x_{s}^{n}, s\right) I_{\left[t_{0}, \tau_{n}\right]} \mathrm{d} s+\int_{t_{0}}^{t} g\left(x_{s}^{n}, s\right) I_{\left[t_{0}, \tau_{n}\right]} \mathrm{d} W(s) \\
& +\int_{t_{0}}^{t} h\left(x_{s}^{n}, s\right) I_{\left[t_{0}, \tau_{n}\right]} \mathrm{d} N(s) .
\end{aligned}
$$


Appling Lemma 3.4 twice and (3.4), one derives that

$$
\begin{aligned}
\left|x^{n}(t)\right|^{2} & \leq \frac{1}{\kappa}\left|G\left(x_{t}^{n}\right)-G(\xi)\right|^{2}+\frac{1}{1-\kappa}\left|J^{n}(t)\right|^{2} \\
& \leq \frac{1}{\kappa} \kappa^{2}\left\|x_{t}^{n}-\xi\right\|^{2}+\frac{1}{1-\kappa}\left|J^{n}(t)\right|^{2} \\
& =\kappa\left\|x_{t}^{n}-\xi\right\|^{2}+\frac{1}{1-\kappa}\left|J^{n}(t)\right|^{2} \\
& \leq \kappa\left(\frac{1}{\sqrt{\kappa}}\left\|x_{t}^{n}\right\|^{2}+\frac{1}{1-\sqrt{\kappa}}\|\xi\|^{2}\right)+\frac{1}{1-\kappa}\left|J^{n}(t)\right|^{2} \\
& =\sqrt{\kappa}\left\|x_{t}^{n}\right\|^{2}+\frac{\kappa}{1-\sqrt{\kappa}}\|\xi\|^{2}+\frac{1}{1-\kappa}\left|J^{n}(t)\right|^{2} .
\end{aligned}
$$

Hence,

$$
\begin{aligned}
E\left(\sup _{t_{0} \leq s \leq t}\left|x^{n}(s)\right|^{2}\right) \leq & \sqrt{\kappa} E\left(\sup _{t_{0}-\tau \leq s \leq t}\left|x^{n}(s)\right|^{2}\right)+\frac{\kappa}{1-\sqrt{\kappa}} E\|\xi\|^{2} \\
& +\frac{1}{1-\kappa} E\left(\sup _{t_{0} \leq s \leq t}\left|J^{n}(s)\right|^{2}\right) .
\end{aligned}
$$

Noting that $E\left(\sup _{t_{0}-\tau \leq s \leq t}\left|x^{n}(s)\right|^{2}\right) \leq E\|\xi\|^{2}+E\left(\sup _{t_{0} \leq s \leq t}\left|x^{n}(s)\right|^{2}\right)$, one sees that

$$
\begin{aligned}
E\left(\sup _{t_{0}-\tau \leq s \leq t}\left|x^{n}(s)\right|^{2}\right) \leq & \sqrt{\kappa} E\left(\sup _{t_{0}-\tau \leq s \leq t}\left|x^{n}(s)\right|^{2}\right)+\frac{1+\kappa-\sqrt{\kappa}}{1-\sqrt{\kappa}} E\|\xi\|^{2} \\
& +\frac{1}{1-\kappa} E\left(\sup _{t_{0} \leq s \leq t}\left|J^{n}(s)\right|^{2}\right) .
\end{aligned}
$$

Consequently,

$$
E\left(\sup _{t_{0}-\tau \leq s \leq t}\left|x^{n}(s)\right|^{2}\right) \leq \frac{1+\kappa-\sqrt{\kappa}}{(1-\sqrt{\kappa})^{2}} E\|\xi\|^{2}+\frac{1}{(1-\kappa)(1-\sqrt{\kappa})} E\left(\sup _{t_{0} \leq s \leq t}\left|J^{n}(s)\right|^{2}\right) .
$$

Using the basic inequality $|a+b+c+d|^{2} \leq 4|a|^{2}+4|b|^{2}+4|c|^{2}+4|d|^{2}$ and the Hölder inequality, and for the jump integral, we can transform to the compensated Poisson process $\widetilde{N}(t)=$ $N(t)-\lambda t$, and we can get

$$
\begin{aligned}
\left|J^{n}(t)\right|^{2} \leq & 4|\xi(0)|^{2}+4\left|\int_{t_{0}}^{t} f\left(x_{s}^{n}, s\right) I_{\left[t_{0}, \tau_{n}\right]}(s) \mathrm{d} s\right|^{2}+4\left|\int_{t_{0}}^{t} g\left(x_{s}^{n}, s\right) I_{\left[t_{0}, \tau_{n}\right]}(s) \mathrm{d} W(s)\right|^{2} \\
& +4\left|\int_{t_{0}}^{t} h\left(x_{s}^{n}, s\right) I_{\left[t_{0}, \tau_{n}\right]}(s) \mathrm{d} N(s)\right|^{2}
\end{aligned}
$$




$$
\begin{aligned}
& \leq 4|\xi(0)|^{2}+4\left(t-t_{0}\right) \int_{t_{0}}^{t}\left|f\left(x_{s}^{n}, s\right)\right|^{2} \mathrm{~d} s+4\left|\int_{t_{0}}^{t} g\left(x_{s}^{n}, s\right) I_{\left[t_{0}, \tau_{n}\right]}(s) \mathrm{d} W(s)\right|^{2} \\
& \quad+4\left|\int_{t_{0}}^{t} h\left(x_{s}^{n}, s\right) I_{\left[t_{0}, \tau_{n}\right]}(s) \mathrm{d} \widetilde{N}(s)+\lambda \int_{t_{0}}^{t} h\left(x_{s}^{n}, s\right) I_{\left[t_{0}, \tau_{n}\right]}(s) \mathrm{d} s\right|^{2} \\
& \leq 4|\xi(0)|^{2}+4\left(t-t_{0}\right) \int_{t_{0}}^{t}\left|f\left(x_{s}^{n}, s\right)\right|^{2} \mathrm{~d} s+4\left|\int_{t_{0}}^{t} g\left(x_{s}^{n}, s\right) I_{\left[t_{0}, \tau_{n}\right]}(s) \mathrm{d} W(s)\right|^{2} \\
& \quad+8\left|\int_{t_{0}}^{t} h\left(x_{s}^{n}, s\right) I_{\left[t_{0}, \tau_{n}\right]}(s) \mathrm{d} \widetilde{N}(s)\right|^{2}+8 \lambda^{2}\left(t-t_{0}\right) \int_{t_{0}}^{t}\left|h\left(x_{s}^{n}, s\right)\right|^{2} \mathrm{~d} s .
\end{aligned}
$$

Since both the Wiener process $W(t)$ and compensated Poisson process $\widetilde{N}(t)$ are martingales, using the Doob martingale inequality, we have

$$
\begin{aligned}
E\left(\sup _{t_{0} \leq s \leq t}\left|J^{n}(s)\right|^{2}\right) \leq & 4 E|\xi(0)|^{2}+4\left(t-t_{0}\right) E \int_{t_{0}}^{t}\left|f\left(x_{s}^{n}, s\right)\right|^{2} \mathrm{~d} s \\
& +4 \times 4 E \int_{t_{0}}^{t}\left|g\left(x_{s}^{n}, s\right)\right|^{2} \mathrm{~d} s \\
& +4\left(2 \times 4 \lambda+2 \lambda^{2}\left(t-t_{0}\right)\right) E \int_{t_{0}}^{t}\left|h\left(x_{s}^{n}, s\right)\right|^{2} \mathrm{~d} s \\
\leq & 4 E\|\xi\|^{2}+4 K\left(T-t_{0}+4+8 \lambda+2 \lambda^{2}\left(T-t_{0}\right)\right) \int_{t_{0}}^{t}\left(1+E\left\|x_{s}^{n}\right\|^{2}\right) \mathrm{d} s .
\end{aligned}
$$

Substituting this into (3.15),

$$
\begin{aligned}
E\left(\sup _{t_{0}-\tau \leq s \leq t}\left|x^{n}(s)\right|^{2}\right) \leq & \frac{5+\kappa \sqrt{\kappa}}{(1-\kappa)(1-\sqrt{\kappa})} E\|\xi\|^{2} \\
& +\frac{4 K\left(T-t_{0}+4+8 \lambda+2 \lambda^{2}\left(T-t_{0}\right)\right)}{(1-\kappa)(1-\sqrt{\kappa})} \int_{t_{0}}^{t}\left(1+E\left\|x_{s}^{n}\right\|^{2}\right) \mathrm{d} s .
\end{aligned}
$$

Therefore,

$$
\begin{aligned}
1+E\left(\sup _{t_{0}-\tau \leq s \leq t}\left|x^{n}(s)\right|^{2}\right) \leq & +\frac{5+\kappa \sqrt{\kappa}}{(1-\kappa)(1-\sqrt{\kappa})} E\|\xi\|^{2} \\
& +\frac{4 K\left(T-t_{0}+4+8 \lambda+2 \lambda^{2}\left(T-t_{0}\right)\right)}{(1-\kappa)(1-\sqrt{\kappa})} \int_{t_{0}}^{t}\left(1+E\left(\sup _{t_{0}-\tau \leq r \leq s}\left|x^{n}(r)\right|^{2}\right)\right) \mathrm{d} s .
\end{aligned}
$$


Now the Gronwall inequality yields that

$$
\begin{aligned}
1+E\left(\sup _{t_{0}-\tau \leq t \leq T}\left|x^{n}(t)\right|^{2}\right) \leq & \left(1+\frac{5+\kappa \sqrt{\kappa}}{(1-\kappa)(1-\sqrt{\kappa})} E\|\xi\|^{2}\right) \\
& \times \exp \left[\frac{4 K\left(T-t_{0}\right)\left(T-t_{0}+4+8 \lambda+2 \lambda^{2}\left(T-t_{0}\right)\right)}{(1-\kappa)(1-\sqrt{\kappa})}\right] .
\end{aligned}
$$

Consequently

$$
\begin{aligned}
E\left(\sup _{t_{0}-\tau \leq t \leq \tau_{n}}|x(t)|^{2}\right) \leq & \left(1+\frac{5+\kappa \sqrt{\kappa}}{(1-\kappa)(1-\sqrt{\kappa})} E\|\xi\|^{2}\right) \\
& \times \exp \left[\frac{4 K\left(T-t_{0}\right)\left(T-t_{0}+4+8 \lambda+2 \lambda^{2}\left(T-t_{0}\right)\right)}{(1-\kappa)(1-\sqrt{\kappa})}\right] .
\end{aligned}
$$

Letting $n \rightarrow \infty$, it then implies the following inequality:

$$
\begin{aligned}
E\left(\sup _{t_{0}-\tau \leq t \leq T}|x(t)|^{2}\right) \leq & \left(1+\frac{5+\kappa \sqrt{\kappa}}{(1-\kappa)(1-\sqrt{\kappa})} E\|\xi\|^{2}\right) \\
& \times \exp \left[\frac{4 K\left(T-t_{0}\right)\left(T-t_{0}+4+8 \lambda+2 \lambda^{2}\left(T-t_{0}\right)\right)}{(1-\kappa)(1-\sqrt{\kappa})}\right] .
\end{aligned}
$$

The proof is complete.

Proof of Theorem 3.2. Uniqueness. Let $x(t)$ and $\bar{x}(t)$ be two solutions to (2.1). By Lemma 3.5 both of them belong to $\mathcal{M}^{2}\left(\left[t_{0}-\tau, T\right] ; R^{n}\right)$. Note that

$$
x(t)-\bar{x}(t)=G\left(x_{t}\right)-G\left(\bar{x}_{t}\right)+J(t)
$$

where

$$
\begin{aligned}
J(t)= & \int_{t_{0}}^{t}\left[f\left(x_{s}, s\right)-f\left(\bar{x}_{s}, s\right)\right] \mathrm{d} s+\int_{t_{0}}^{t}\left[g\left(x_{s}, s\right)-g\left(\bar{x}_{s}, s\right)\right] \mathrm{d} W(s) \\
& +\int_{t_{0}}^{t}\left[h\left(x_{s}, s\right)-h\left(\bar{x}_{s}, s\right)\right] \mathrm{d} N(s)
\end{aligned}
$$

By Lemma 3.4 and condition (3.4), one sees easily that

$$
\begin{aligned}
|x(t)-\bar{x}(t)|^{2} & \leq \frac{1}{\kappa}\left|G\left(x_{t}\right)-G\left(\bar{x}_{t}\right)\right|^{2}+\frac{1}{1-\kappa}|J(t)|^{2} \\
& \leq \kappa\left\|x_{t}-\bar{x}_{t}\right\|^{2}+\frac{1}{1-\kappa}|J(t)|^{2} .
\end{aligned}
$$


Therefore,

$$
E \sup _{t_{0} \leq s \leq t}|x(s)-\bar{x}(s)|^{2} \leq \kappa E \sup _{t_{0} \leq s \leq t}|x(s)-\bar{x}(s)|^{2}+\frac{1}{1-\kappa} E \sup _{t_{0} \leq s \leq t}|J(s)|^{2},
$$

which implies that

$$
E \sup _{t_{0} \leq s \leq t}|x(s)-\bar{x}(s)|^{2} \leq \frac{1}{(1-\kappa)^{2}} E \sup _{t_{0} \leq s \leq t}|J(s)|^{2}
$$

On the other hand,

$$
\begin{aligned}
E \sup _{t_{0} \leq s \leq t}|J(s)|^{2} & \leq 3 \bar{K}\left(T-t_{0}+4+8 \lambda+2 \lambda^{2}\left(T-t_{0}\right)\right) \int_{t_{0}}^{t} E\left\|x_{s}-\bar{x}_{s}\right\|^{2} \mathrm{~d} s \\
& =3 \bar{K}\left(T-t_{0}+4+8 \lambda+2 \lambda^{2}\left(T-t_{0}\right)\right) \int_{t_{0}}^{t} E \sup _{t_{0}-\tau \leq r \leq s}|x(r)-\bar{x}(r)|^{2} \mathrm{~d} s .
\end{aligned}
$$

Noting the fact that $x(r)=\bar{x}(r)=\xi$, for $r \in\left[t_{0}-\tau, t_{0}\right]$, we have

$$
\sup _{t_{0}-\tau \leq r \leq s}|x(r)-\bar{x}(r)|^{2}=\sup _{t_{0} \leq r \leq s}|x(r)-\bar{x}(r)|^{2} .
$$

Then

$$
E \sup _{t_{0} \leq s \leq t}|J(s)|^{2} \leq 3 \bar{K}\left(T-t_{0}+4+8 \lambda+2 \lambda^{2}\left(T-t_{0}\right)\right) \int_{t_{0}}^{t} E \sup _{t_{0} \leq r \leq s}|x(r)-\bar{x}(r)|^{2} \mathrm{~d} s .
$$

Therefore,

$$
\begin{aligned}
E \sup _{t_{0} \leq s \leq t} & |x(s)-\bar{x}(s)|^{2} \\
& \leq \frac{3 \bar{K}\left(T-t_{0}+4+8 \lambda+2 \lambda^{2}\left(T-t_{0}\right)\right)}{(1-\kappa)^{2}} \int_{t_{0}}^{t} E \sup _{t_{0} \leq r \leq s}|x(r)-\bar{x}(r)|^{2} \mathrm{~d} s .
\end{aligned}
$$

The Grownwall inequality then yields that

$$
E \sup _{t_{0} \leq t \leq T}|x(t)-\bar{x}(t)|^{2}=0 .
$$

Therefore the uniqueness has been proved.

Existence. We divide the whole proof of the existence into two steps.

Step 1 . We impose an additional condition: $T-t_{0}$ is sufficiently small so that

$$
\delta:=\kappa+\frac{3 \bar{K}\left(T-t_{0}+4+8 \lambda+2 \lambda^{2}\left(T-t_{0}\right)\right)}{1-\kappa}\left(T-t_{0}\right)<1 .
$$


Define $x_{t_{0}}^{0}=\xi$ and $x^{0}(t)=\xi(0)$ for $t_{0} \leq t \leq T$. For each $n=1,2, \ldots$, set $x_{t_{0}}^{n}=\xi$ and define, by the Picard iterations

$$
\begin{aligned}
x^{n}(t)-G\left(x_{t}^{n-1}\right)= & \xi(0)-G(\xi)+\int_{t_{0}}^{t} f\left(x_{s}^{n-1}, s\right) \mathrm{d} s+\int_{t_{0}}^{t} g\left(x_{s}^{n-1}, s\right) \mathrm{d} W(s) \\
& +\int_{t_{0}}^{t} h\left(x_{s}^{n-1}, s\right) \mathrm{d} N(s) .
\end{aligned}
$$

Obviously, $x^{0}(t) \in \mathcal{M}^{2}\left(\left[t_{0}-\tau, T\right] ; R^{n}\right)$, and by induction $x^{n}(t) \in \mathcal{M}^{2}\left(\left[t_{0}-\tau, T\right] ; R^{n}\right)$. In fact,

$$
\begin{aligned}
x^{n}(t)= & G\left(x_{t}^{n-1}\right)-G(\xi)+\xi(0)+\int_{t_{0}}^{t} f\left(x_{s}^{n-1}, s\right) \mathrm{d} s+\int_{t_{0}}^{t} g\left(x_{s}^{n-1}, s\right) \mathrm{d} W(s) \\
& +\int_{t_{0}}^{t} h\left(x_{s}^{n-1}, s\right) \mathrm{d} N(s) .
\end{aligned}
$$

Let

$$
P^{n-1}(t)=\xi(0)+\int_{t_{0}}^{t} f\left(x_{s}^{n-1}, s\right) \mathrm{d} s+\int_{t_{0}}^{t} g\left(x_{s}^{n-1}, s\right) \mathrm{d} W(s)+\int_{t_{0}}^{t} h\left(x_{s}^{n-1}, s\right) \mathrm{d} N(s) .
$$

Then by Lemma 3.4 and (3.4) we have

$$
\begin{aligned}
\left|x^{n}(t)\right|^{2} & \leq \frac{1}{\kappa}\left|G\left(x_{t}^{n}\right)-G(\xi)\right|^{2}+\frac{1}{1-\kappa}\left|P^{n-1}(t)\right|^{2} \\
& \leq \kappa\left\|x_{t}^{n}-\xi\right\|^{2}+\frac{1}{1-\kappa}\left|P^{n-1}(t)\right|^{2} \\
& \leq \kappa\left(\frac{1}{\sqrt{\kappa}}\left\|x_{t}^{n}\right\|^{2}+\frac{1}{1-\sqrt{\kappa}}\|\xi\|^{2}\right)+\frac{1}{1-\kappa}\left|P^{n-1}(t)\right|^{2} \\
& =\sqrt{\kappa}\left\|x_{t}^{n}\right\|^{2}+\frac{\kappa}{1-\sqrt{\kappa}}\|\xi\|^{2}+\frac{1}{1-\kappa}\left|P^{n-1}(t)\right|^{2} \\
& \leq \sqrt{\kappa}\left(\|\xi\|^{2}+\left|x^{n}(t)\right|^{2}\right)+\frac{\kappa}{1-\sqrt{\kappa}}\|\xi\|^{2}+\frac{1}{1-\kappa}\left|P^{n-1}(t)\right|^{2} .
\end{aligned}
$$

Then,

$$
\left|x^{n}(t)\right|^{2} \leq \frac{\sqrt{\kappa}}{(1-\sqrt{\kappa})^{2}}\|\xi\|^{2}+\frac{1}{(1-\sqrt{\kappa})(1-\kappa)}\left|P^{n-1}(t)\right|^{2}
$$

Therefore,

$$
E\left|x^{n}(t)\right|^{2} \leq \frac{\sqrt{\kappa}}{(1-\sqrt{\kappa})^{2}} E\|\xi\|^{2}+\frac{1}{(1-\sqrt{\kappa})(1-\kappa)} E\left|P^{n-1}(t)\right|^{2}
$$


By the basic inequality $|a+b+c+d|^{2} \leq 4|a|^{2}+4|b|^{2}+4|c|^{2}+4|d|^{2}$, the Hölder inequality, Lemma 3.3, and martingale isometry, we have

$$
\begin{aligned}
E\left|P^{n-1}(t)\right|^{2} \leq & 4 E|\xi(0)|^{2}+4 E\left|\int_{t_{0}}^{t} f\left(x_{s}^{n-1}, s\right) \mathrm{d} s\right|^{2} \\
& +4 E\left|\int_{t_{0}}^{t} g\left(x_{s}^{n-1}, s\right) \mathrm{d} W(s)\right|^{2}+4 E\left|\int_{t_{0}}^{t} h\left(x_{s}^{n-1}, s\right) \mathrm{d} N(s)\right|^{2} \\
\leq & 4 E|\xi(0)|^{2}+4\left(t-t_{0}\right) E \int_{t_{0}}^{t}\left|f\left(x_{s}^{n-1}, s\right)\right|^{2} \mathrm{~d} s+4 E \int_{t_{0}}^{t}\left|g\left(x_{s}^{n-1}, s\right)\right|^{2} \mathrm{~d} s \\
& +4\left(2 \lambda+2 \lambda^{2}\left(t-t_{0}\right)\right) E \int_{t_{0}}^{t}\left|h\left(x_{s}^{n-1}, s\right)\right|^{2} \mathrm{~d} s \\
\leq & 4 E\|\xi\|^{2}+4 K\left(T-t_{0}+1\right) \int_{t_{0}}^{t}\left(1+E\left\|x_{s}^{n-1}\right\|^{2}\right) \mathrm{d} s \\
& +4 K\left(2 \lambda+2 \lambda^{2}\left(T-t_{0}\right)\right) \int_{t_{0}}^{t}\left(1+E\left\|x_{s}^{n-1}\right\|^{2}\right) \mathrm{d} s \\
\leq & 4 E\|\xi\|^{2}+4 K\left(T-t_{0}+1+2 \lambda+2 \lambda^{2}\left(T-t_{0}\right)\right)\left(T-t_{0}\right) \\
& +4 K\left(T-t_{0}+1+2 \lambda+2 \lambda^{2}\left(T-t_{0}\right)\right) E \int_{t_{0}}^{t} \sup t_{0} \leq r \leq s \\
& +4 K\left(T-\left.x_{0}^{n-1}(r)\right|^{2} \mathrm{~d} s\right. \\
\leq & 4 E\|\xi\|^{2}+4 K\left(T-t_{0}+1+2 \lambda+2 \lambda^{2}\left(T-t_{0}\right)\right)\left(T-t_{0}\right) \\
& \\
& \\
& \\
& \\
&
\end{aligned}
$$

Therefore,

$$
\begin{aligned}
E\left|x^{n}(t)\right|^{2} \leq & \frac{\sqrt{\kappa}}{(1-\sqrt{\kappa})^{2}} E\|\xi\|^{2}+\frac{1}{(1-\sqrt{\kappa})(1-\kappa)} E\left|P^{n-1}(t)\right|^{2} \\
\leq & \frac{\sqrt{\kappa}}{(1-\sqrt{\kappa})^{2}} E\|\xi\|^{2}+\frac{4}{(1-\sqrt{\kappa})(1-\kappa)} E\|\xi\|^{2} \\
& +\frac{4 K\left(T-t_{0}+1+2 \lambda+2 \lambda^{2}\left(T-t_{0}\right)\right)\left(T-t_{0}\right)}{(1-\sqrt{\kappa})(1-\kappa)} \\
& +\frac{4 K\left(T-t_{0}+1+2 \lambda+2 \lambda^{2}\left(T-t_{0}\right)\right)}{(1-\sqrt{\kappa})(1-\kappa)} \int_{t_{0}}^{t} E\left|x^{n-1}(s)\right|^{2} \mathrm{~d} s .
\end{aligned}
$$


Hence, for any $k \geq 1$, we have

$$
\begin{aligned}
\max _{1 \leq n \leq k} E\left|x^{n}(t)\right|^{2} \leq & {\left[\frac{\sqrt{\kappa}}{(1-\sqrt{\kappa})^{2}}+\frac{4}{(1-\sqrt{\kappa})(1-\kappa)}\right] E\|\xi\|^{2} } \\
& +\frac{4 K\left(T-t_{0}+1+2 \lambda+2 \lambda^{2}\left(T-t_{0}\right)\right)\left(T-t_{0}\right)}{(1-\sqrt{\kappa})(1-\kappa)} \\
& +\frac{4 K\left(T-t_{0}+1+2 \lambda+2 \lambda^{2}\left(T-t_{0}\right)\right)}{(1-\sqrt{\kappa})(1-\kappa)} \int_{t_{0}}^{t} \max _{1 \leq n \leq k} E\left|x^{n-1}(s)\right|^{2} \mathrm{~d} s .
\end{aligned}
$$

Note that

$$
\begin{aligned}
\max _{1 \leq n \leq k} E\left|x^{n-1}(t)\right|^{2} & =\max \left\{E|\xi(0)|^{2}, E\left|x^{1}(s)\right|^{2}, \ldots, E\left|x^{k-1}(s)\right|^{2}\right\} \\
& \leq \max \left\{E\|\xi\|^{2}, E\left|x^{1}(s)\right|^{2}, \ldots, E\left|x^{k-1}(s)\right|^{2}\right\} \\
& =\max \left\{E\|\xi\|^{2}, \max _{1 \leq n \leq k} E\left|x^{n}(t)\right|^{2}\right\} \\
& \leq E\|\xi\|^{2}+\max _{1 \leq n \leq k} E\left|x^{n}(t)\right|^{2} .
\end{aligned}
$$

Therefore,

$$
\begin{aligned}
\max _{1 \leq n \leq k} E\left|x^{n}(t)\right|^{2} \leq & {\left[\frac{\sqrt{\kappa}}{(1-\sqrt{\kappa})^{2}}+\frac{4}{(1-\sqrt{\kappa})(1-\kappa)}\right] E\|\xi\|^{2} } \\
& +\frac{4 K\left(T-t_{0}+1+2 \lambda+2 \lambda^{2}\left(T-t_{0}\right)\right)\left(T-t_{0}\right)}{(1-\sqrt{\kappa})(1-\kappa)} \\
& +\frac{4 K\left(T-t_{0}+1+2 \lambda+2 \lambda^{2}\left(T-t_{0}\right)\right)}{(1-\sqrt{\kappa})(1-\kappa)} \int_{t_{0}}^{t}\left(E\|\xi\|^{2}+\max _{1 \leq n \leq k} E\left|x^{n}(t)\right|^{2}\right) \mathrm{d} s \\
\leq & c_{1}+c_{2} \int_{t_{0}}^{t} \max _{1 \leq n \leq k} E\left|x^{n}(s)\right|^{2} \mathrm{~d} s,
\end{aligned}
$$

where $c_{1}=\left[\left(\sqrt{\kappa} /(1-\sqrt{\kappa})^{2}\right)+(4 /(1-\sqrt{\kappa})(1-\kappa))+\left(4 K\left(T-t_{0}+1+2 \lambda+2 \lambda^{2}\left(T-t_{0}\right)\right) /((1-\right.\right.$ $\left.\sqrt{\kappa})(1-\kappa))) E\|\xi\|^{2}\right] E\|\xi\|^{2}+\left(4 K\left(T-t_{0}+1+2 \lambda+2 \lambda^{2}\left(T-t_{0}\right)\right)\left(T-t_{0}\right) /((1-\sqrt{\kappa})(1-\kappa))\right), c_{2}=$ $\left(4 K\left(T-t_{0}+1+2 \lambda+2 \lambda^{2}\left(T-t_{0}\right)\right) /((1-\sqrt{\kappa})(1-\kappa))\right)$.

By the Gronwall inequality, we have

$$
\max _{1 \leq n \leq k} E\left|x^{n}(t)\right|^{2} \leq c_{1} e^{c_{2}\left(T-t_{0}\right)} .
$$

Because $k$ is arbitrary, we have

$$
E\left|x^{n}(t)\right|^{2} \leq c_{1} e^{c_{2}\left(T-t_{0}\right)} .
$$

Hence, for all $n \geq 0, x^{n}(t) \in \mathcal{M}^{2}\left(\left[t_{0}-\tau, T\right] ; R^{n}\right)$. 
Note that, for $t_{0} \leq t \leq T$,

$$
\begin{aligned}
x^{1}(t)-x^{0}(t)= & x^{1}(t)-\xi(0) \\
= & G\left(x_{t}^{0}\right)-G(\xi)+\int_{t_{0}}^{t} f\left(x_{s}^{0}, s\right) \mathrm{d} s+\int_{t_{0}}^{t} g\left(x_{s}^{0}, s\right) \mathrm{d} W(s) \\
& +\int_{t_{0}}^{t} h\left(x_{s}^{0}, s\right) \mathrm{d} N(s) .
\end{aligned}
$$

In a similar way to that in the proof of the existence, we get that

$$
\begin{aligned}
E \sup _{t_{0} \leq t \leq T}\left|x^{1}(t)-x^{0}(t)\right|^{2} \leq & \kappa E \sup _{t_{0} \leq t \leq T}\left\|x_{t}^{0}-\xi\right\|^{2} \\
& +\frac{3 K\left(T-t_{0}+4+8 \lambda+2 \lambda^{2}\left(T-t_{0}\right)\right)}{1-\kappa} \int_{t_{0}}^{t}\left(1+\left\|x_{t}^{0}\right\|\right)^{2} \mathrm{~d} s \\
\leq & 2 \kappa E\|\xi\|^{2}+\frac{3 K\left(T-t_{0}+4+8 \lambda+2 \lambda^{2}\left(T-t_{0}\right)\right)}{1-\kappa}\left(1+E\|\xi\|^{2}\right)\left(T-t_{0}\right):=C .
\end{aligned}
$$

Note also that, for $n \geq 1$ and $t_{0} \leq t \leq T$,

$$
\begin{aligned}
x^{n+1}(t)-x^{n}(t)= & G\left(x_{t}^{n}\right)-G\left(x_{t}^{n-1}\right)+\int_{t_{0}}^{t}\left[f\left(x_{s}^{n}, s\right)-f\left(x_{s}^{n-1}, s\right)\right] \mathrm{d} s \\
& +\int_{t_{0}}^{t}\left[g\left(x_{s}^{n}, s\right)-g\left(x_{s}^{n-1}, s\right)\right] \mathrm{d} W(s) \\
& +\int_{t_{0}}^{t}\left[h\left(x_{s}^{n}, s\right)-h\left(x_{s}^{n-1}, s\right)\right] \mathrm{d} N(s) .
\end{aligned}
$$

In the same way as in the proof of the uniqueness, one derives that

$$
\begin{aligned}
E \sup _{t_{0} \leq t \leq T} & \left|x^{n+1}(t)-x^{n}(t)\right|^{2} \\
\leq & \kappa E \sup _{t_{0} \leq t \leq T}\left|x^{n}(t)-x^{n-1}(t)\right|^{2} \\
& +\frac{3 \bar{K}\left(T-t_{0}+4+8 \lambda+2 \lambda^{2}\left(T-t_{0}\right)\right)}{1-\kappa} \int_{t_{0}}^{T} E \sup _{t_{0} \leq s \leq t}\left|x^{n}(s)-x^{n-1}(s)\right|^{2} \mathrm{~d} t \\
& \leq \delta E \sup _{t_{0} \leq t \leq T}\left|x^{n}(t)-x^{n-1}(t)\right|^{2}
\end{aligned}
$$




$$
\begin{aligned}
& \leq \delta^{n} E \sup _{t_{0} \leq t \leq T}\left|x^{1}(t)-x^{0}(t)\right|^{2} \\
& \leq C \delta^{n} .
\end{aligned}
$$

Next we verify that $\left\{x^{n}(t)\right\}$ converges to $x(t)$ in the sense of $L^{2}$ and probability 1 on $\mathcal{M}^{2}\left(\left[t_{0}-\tau, T\right] ; R^{n}\right)$. Moreover, $x(t)$ is the solution to (2.1) with initial data (2.3). Hence, by the Chebyshev inequality,

$$
P\left\{\sup _{t_{0} \leq t \leq T}\left|x^{n+1}(t)-x^{n}(t)\right|^{2}>\frac{1}{2^{n}}\right\} \leq C(4 \delta)^{n}
$$

Since $\sum_{n=0}^{\infty} C(4 \delta)^{n} \leq \infty$, the Borel-Cantelli lemma yields that, for almost all $\omega \in \Omega$, there exist a positive integer $n_{0}=n_{0}(\omega)$ such that

$$
\sup _{t_{0} \leq t \leq T}\left|x^{n+1}(t)-x^{n}(t)\right|^{2} \leq \frac{1}{2^{n}}, \quad n \geq n_{0}
$$

It follows that, with probability 1 , the partial sum

$$
x^{0}(t)+\sum_{i=0}^{n-1}\left[x^{i+1}(t)-x^{i}(t)\right]=x^{n}(t)
$$

is the partial sum of function series

$$
x^{0}(t)+\left|x^{1}(t)-x^{0}(t)\right|+\cdots+\left|x^{n}(t)-x^{n-1}(t)\right|+\cdots
$$

By the second item of series (3.54), the absolute value of every item (3.54) is less than the corresponding item of positive

$$
1+\frac{1}{2}+\frac{1}{2^{2}}+\cdots+\frac{1}{2^{n}}+\cdots .
$$

Moreover, the positive series is convergent; further, by the Weierstrass criterion, series (3.54) is convergent on $\left[t_{0}, T\right]$. Furthermore, it is uniformly on $\left[t_{0}, T\right]$. Let the sum function be $x(t)$. Therefore, the approximate sequence $\left\{x^{n}(t)\right\}$ uniformly converges to $x(t)$ on $\left[t_{0}, T\right]$ and is $\mathcal{F}_{t}$-adapted, hence $x(t)$ is also continuous and $\mathcal{F}_{t}$-adapted. On the other hand, (3.50) implies that, for each $t$, sequence $\left\{x^{n}(t)\right\}$ is a Cauchy sequence in $\mathcal{M}^{2}\left(\left[t_{0}-\tau, T\right] ; R^{n}\right)$ as well. Hence, we also have $x^{n}(t) \rightarrow x(t)$ in $\mathcal{M}^{2}\left(\left[t_{0}-\tau, T\right] ; R^{n}\right)$. Letting $n \rightarrow \infty$ in (3.46) gives

$$
E|x(t)|^{2} \leq c_{1} e^{c_{2}\left(T-t_{0}\right)}, \quad t_{0} \leq t \leq T .
$$


Therefore, by the use of the above result, we get that

$$
\begin{aligned}
E \int_{t_{0}-\tau}^{T}|x(s)|^{2} \mathrm{~d} s & =E \int_{t_{0}-\tau}^{t_{0}}|x(s)|^{2} \mathrm{~d} s+E \int_{t_{0}}^{T}|x(s)|^{2} \mathrm{~d} s \\
& \leq E \int_{t_{0}-\tau}^{t_{0}}|\xi(s)|^{2} \mathrm{~d} s+E \int_{t_{0}-\tau}^{T} c_{1} e^{c_{2}\left(T-t_{0}\right)} \mathrm{d} s<\infty .
\end{aligned}
$$

That is, $x(t) \in \mathcal{M}^{2}\left(\left[t_{0}-\tau, T\right] ; R^{n}\right)$.

Now we prove that $x(t)$ satisfies (2.1):

$$
\begin{aligned}
E\left|\int_{t_{0}}^{t}\left[f\left(x_{s}^{n}, s\right)-f\left(x_{s}, s\right)\right] \mathrm{d} s\right|^{2} & +E\left|\int_{t_{0}}^{t}\left[g\left(x_{s}^{n}, s\right)-g\left(x_{s}, s\right)\right] \mathrm{d} W(s)^{2}\right| \\
& +E\left|\int_{t_{0}}^{t}\left[h\left(x_{s}^{n}, s\right)-h\left(x_{s}, s\right)\right] \mathrm{d} N(s)\right|^{2} \\
\leq & \left(t-t_{0}\right) E \int_{t_{0}}^{t}\left|f\left(x_{s}^{n}, s\right)-f\left(x_{s}, s\right)\right|^{2} \mathrm{~d} s \\
& +E \int_{t_{0}}^{t}\left|g\left(x_{s}^{n}, s\right)-g\left(x_{s}, s\right)\right|^{2} \mathrm{~d} s \\
& +\left(2 \lambda+2 \lambda^{2}\left(t-t_{0}\right)\right) E \int_{t_{0}}^{t}\left|h\left(x_{s}^{n}, s\right)-h\left(x_{s}, s\right)\right|^{2} \mathrm{~d} s \\
\leq & \left(t-t_{0}\right) \bar{K} E \int_{t_{0}}^{t}\left\|x_{s}^{n}-x_{s}\right\|^{2} \mathrm{~d} s+\bar{K} E \int_{t_{0}}^{t}\left\|x_{s}^{n}-x_{s}\right\|^{2} \mathrm{~d} s \\
& +\left(2 \lambda+2 \lambda^{2}\left(t-t_{0}\right)\right) \bar{K} E \int_{t_{0}}^{t}\left\|x_{s}^{n}-x_{s}\right\| \mathrm{d} s \\
\leq & \bar{K}\left(T-t_{0}+1+2 \lambda+2 \lambda^{2}\left(T-t_{0}\right)\right) E \int_{t_{0}}^{t}\left\|x_{s}^{n}-x_{s}\right\| \mathrm{d} s \\
\leq & \bar{K}\left(T-t_{0}+1+2 \lambda+2 \lambda^{2}\left(T-t_{0}\right)\right) \int_{t_{0}}^{t} E t_{0} \leq r \leq s \\
\leq & \left.\left(T-t_{0}+1+2 \lambda+2 \lambda^{2}\left(T-t_{0}\right)\right) \int_{t_{0}}^{T} E\left|x^{n}(s)-x(s)\right|^{2} \mathrm{~d} s \longrightarrow 0\right) . \\
& \\
\leq & \left.(r)\right|^{2} \mathrm{~d} s \\
& \\
& \\
& \\
&
\end{aligned}
$$


Hence, we can let $n \rightarrow \infty$ in (3.34) to obtain that

$$
\begin{aligned}
x(t)-G\left(x_{t}\right)= & \xi(0)-G(\xi)+\int_{t_{0}}^{t} f\left(x_{s}, s\right) \mathrm{d} s+\int_{t_{0}}^{t} g\left(x_{s}, s\right) \mathrm{d} W(s) \\
& +\int_{t_{0}}^{t} h\left(x_{s}, s\right) \mathrm{d} N(s) .
\end{aligned}
$$

So $x(t)$ is the solution to (2.1).

Step 2. We need to remove the additional condition (3.33). Let $\sigma>0$ be sufficiently small for

$$
\delta:=\kappa+\frac{3 \bar{K}\left(\sigma+4+8 \lambda+2 \lambda^{2} \sigma\right)}{1-\kappa} \sigma<1
$$

By Step 1, there is a solution to (2.1) on $\left[t_{0}-\tau, t_{0}+\sigma\right]$. Now consider (2.1) on $\left[t_{0}+\sigma, t_{0}+2 \sigma\right]$ with initial data $x_{t_{0}+\sigma}$. By Step 1 again, there is a solution to (2.1) on $\left[t_{0}+\sigma, t_{0}+2 \sigma\right]$. Repeating this procedure we see that there is a solution to (2.1) on the entire interval $\left[t_{0}-\tau, T\right]$. The proof is complete.

For NSFDEwPJs, we know that the global Lipschitz condition imposed on Theorem 3.2 is a big restriction; now we will replace the global Lipschitz condition by the local Lipschitz condition. Then Theorem 3.6 follows.

Theorem 3.6. Assume that there exist two positive constants $K_{n}$ and $K$ such that

(i) (the local Lipschitz condition) for all $\phi, \varphi \in C\left([-\tau, 0] ; R^{n}\right)$ with $\|\phi\| \vee \mid \varphi \| \leq n$ and $t \in$ $\left[t_{0}, T\right]$,

$$
\begin{aligned}
& |f(\phi, t)-f(\varphi, t)|^{2} \vee|g(\phi, t)-g(\varphi, t)|^{2} \vee|h(\phi, t)-h(\varphi, t)|^{2} \\
& \quad \leq K_{n}\|\phi-\varphi\|^{2} .
\end{aligned}
$$

(ii) (the linear growth condition) for all $(\phi, t) \in C\left([-\tau, 0] ; R^{n}\right) \times\left[t_{0}, T\right]$,

$$
|f(\phi, t)|^{2} \vee|g(\phi, t)|^{2} \vee|h(\phi, t)|^{2} \leq K\left(1+\|\phi\|^{2}\right) .
$$

(iii) (the constractive mapping) there is a positive constant $\kappa \in(0,1)$ such that, for all $\phi, \varphi \in$ $C\left([-\tau, 0] ; R^{n}\right)$,

$$
|G(\phi)-G(\varphi)| \leq \kappa\|\phi-\varphi\|
$$

Then there exists a unique solution $x(t)$ to (2.1) with initial data (2.3). Moreover, the solution belongs to $\mathcal{M}^{2}\left(\left[t_{0}-\tau, T\right] ; R^{n}\right)$. 
Proof. For each $n \geq 1$, define truncation functions $f_{n}, g_{n}$, and $h_{n}$ as follows:

$$
\begin{aligned}
& f_{n}\left(x_{t}, t\right)= \begin{cases}f\left(x_{t}, t\right) & \left\|x_{t}\right\| \leq n, \\
f\left(\frac{n x_{t}}{\left\|x_{t}\right\|}, t\right) & \left\|x_{t}\right\|>n,\end{cases} \\
& g_{n}\left(x_{t}, t\right)= \begin{cases}g\left(x_{t}, t\right) & \left\|x_{t}\right\| \leq n, \\
g\left(\frac{n x_{t}}{\left\|x_{n}\right\|}, t\right) & \left\|x_{t}\right\|>n,\end{cases} \\
& h_{n}\left(x_{t}, t\right)= \begin{cases}h\left(x_{t}, t\right) \\
h\left(\frac{n x_{t}}{\left\|x_{n}\right\|}, t\right) & \left\|x_{t}\right\|>n,\end{cases}
\end{aligned}
$$

Then $f_{n}, g_{n}$, and $h_{n}$ satisfy conditions (3.2) and (3.3). By Theorem 3.2, there is a unique solution $x^{n}(t)$ and $x^{n}(t) \in \mathcal{M}^{2}\left(\left[t_{0}-\tau, T\right] ; R^{n}\right)$ to the equation

$$
\begin{aligned}
x^{n}(t)-G\left(x_{t}^{n}\right)= & \xi(0)-G(\xi)+\int_{t_{0}}^{t} f_{n}\left(x_{s}^{n}, s\right) \mathrm{d} s \\
& +\int_{t_{0}}^{t} g_{n}\left(x_{s}^{n}, s\right) \mathrm{d} W(s) \\
& +\int_{t_{0}}^{t} h_{n}\left(x_{s}^{n}, s\right) \mathrm{d} N(s), \quad t \in\left[t_{0}, T\right] .
\end{aligned}
$$

Certainly $x^{n+1}(t)$ is a unique solution to the equation

$$
\begin{aligned}
x^{n+1}(t)-G\left(x_{t}^{n+1}\right)= & \xi(0)-G(\xi)+\int_{t_{0}}^{t} f_{n+1}\left(x_{s}^{n+1}, s\right) \mathrm{d} s \\
& +\int_{t_{0}}^{t} g_{n+1}\left(x_{s}^{n+1}, s\right) \mathrm{d} W(s) \\
& +\int_{t_{0}}^{t} h_{n+1}\left(x_{s}^{n+1}, s\right) \mathrm{d} N(s), \quad t \in\left[t_{0}, T\right]
\end{aligned}
$$

and $x^{n+1}(t) \in \mathcal{M}^{2}\left(\left[t_{0}-\tau, T\right] ; R^{n}\right)$ to the equation.

Now define the stopping time

$$
\tau_{n}=T \wedge \inf \left\{t \in\left[t_{0}, T\right]:\left\|x_{t}^{n}\right\| \geq n\right\}
$$


We can show that $x^{n}(t)=x^{n+1}(t)$ for $t \in\left[t_{0}, T\right]$. Then by Lemma 3.4, basic inequality, we have

$$
\begin{aligned}
\left|x^{n+1}(t)-x^{n}(t)\right|^{2} \leq & \frac{1}{\mathcal{\kappa}}\left|G\left(x_{t}^{n+1}\right)-G\left(x_{t}^{n}\right)\right|^{2} \\
& +\frac{3}{1-\kappa}\left|\int_{t_{0}}^{t}\left[f_{n+1}\left(x_{s}^{n+1}, s\right)-f_{n}\left(x_{s}^{n}, s\right)\right] \mathrm{d} s\right|^{2} \\
& +\frac{3}{1-\kappa}\left|\int_{t_{0}}^{t}\left[g_{n+1}\left(x_{s}^{n+1}, s\right)-g_{n}\left(x_{s}^{n}, s\right)\right] \mathrm{d} W(s)\right|^{2} \\
& +\frac{3}{1-\kappa}\left|\int_{t_{0}}^{t}\left[h_{n+1}\left(x_{s}^{n+1}, s\right)-h_{n}\left(x_{s}^{n}, s\right)\right] \mathrm{d} N(s)\right|^{2} .
\end{aligned}
$$

Taking the expectation, by the Hölder inequality and Lemma 3.3, we transform the Poisson process to the compensated Poisson process $\widetilde{N}(t)=N(t)-\lambda t$, and, by martingale isometry, we have

$$
\begin{aligned}
E \mid x^{n+1}(t) & -\left.x^{n}(t)\right|^{2} \\
\leq & \kappa E|| x_{t}^{n+1}-x_{t}^{n} \|^{2}+\frac{3}{1-\kappa}\left(t-t_{0}\right) E \int_{t_{0}}^{t}\left|f_{n+1}\left(x_{s}^{n+1}, s\right)-f_{n}\left(x_{s}^{n}, s\right)\right|^{2} \mathrm{~d} s \\
& +\frac{3}{1-\mathcal{K}} E \int_{t_{0}}^{t}\left|g_{n+1}\left(x_{s}^{n+1}, s\right)-g_{n}\left(x_{s}^{n}, s\right)\right|^{2} \mathrm{~d} s \\
& +\frac{3}{1-\mathcal{\kappa}}\left(2 \lambda+2 \lambda^{2}\left(t-t_{0}\right)\right) E \int_{t_{0}}^{t}\left|h_{n+1}\left(x_{s}^{n+1}, s\right)-h_{n}\left(x_{s}^{n}, s\right)\right|^{2} \mathrm{~d} s \\
\leq & \kappa E\left\|x_{t}^{n+1}-x_{t}^{n}\right\|^{2}+\frac{6}{1-\mathcal{\kappa}}\left(t-t_{0}\right) E \int_{t_{0}}^{t}\left|f_{n+1}\left(x_{s}^{n+1}, s\right)-f_{n+1}\left(x_{s}^{n}, s\right)\right|^{2} \mathrm{~d} s \\
& +\frac{6}{1-\mathcal{K}}\left(t-t_{0}\right) E \int_{t_{0}}^{t}\left|f_{n+1}\left(x_{s}^{n}, s\right)-f_{n}\left(x_{s}^{n}, s\right)\right|^{2} \mathrm{~d} s \\
& +\frac{6}{1-\mathcal{K}} E \int_{t_{0}}^{t}\left|g_{n+1}\left(x_{s}^{n+1}, s\right)-g_{n+1}\left(x_{s}^{n}, s\right)\right|^{2} \mathrm{~d} s \\
& +\frac{6}{1-\mathcal{K}} E \int_{t_{0}}^{t}\left|g_{n+1}\left(x_{s}^{n}, s\right)-g_{n}\left(x_{s}^{n}, s\right)\right|^{2} \mathrm{~d} s \\
& +\frac{6}{1-\mathcal{K}}\left(2 \lambda+2 \lambda^{2}\left(t-t_{0}\right)\right) E \int_{t_{0}}^{t}\left|h_{n+1}\left(x_{s}^{n+1}, s\right)-h_{n+1}\left(x_{s}^{n}, s\right)\right|^{2} \mathrm{~d} s \\
& +\frac{6}{1-\mathcal{K}}\left(2 \lambda+2 \lambda^{2}\left(t-t_{0}\right)\right) E \int_{t_{0}}^{t}\left|h_{n+1}\left(x_{s}^{n}, s\right)-h_{n}\left(x_{s}^{n}, s\right)\right|^{2} \mathrm{~d} s .
\end{aligned}
$$


For $t_{0}<t<t_{n}$, we have know that

$$
\begin{aligned}
& f_{n+1}\left(x_{s}^{n}, s\right)=f_{n}\left(x_{s}^{n}, s\right)=f\left(x_{s}^{n}, s\right), \\
& g_{n+1}\left(x_{s}^{n}, s\right)=g_{n}\left(x_{s}^{n}, s\right)=g\left(x_{s}^{n}, s\right), \\
& h_{n+1}\left(x_{s}^{n}, s\right)=h_{n}\left(x_{s}^{n}, s\right)=h\left(x_{s}^{n}, s\right) .
\end{aligned}
$$

Again by $x_{t_{0}}^{n+1}=x_{t_{0}}^{n}=\xi$, we get that

$$
\begin{aligned}
E\left|x^{n+1}(s)-x^{n}(s)\right|^{2} & \\
\leq & \kappa E \| x_{t}^{n+1}-\left.x_{t}^{n}\right|^{2}+\frac{6}{1-\kappa}\left(t-t_{0}\right) E \int_{t_{0}}^{t}\left|f_{n+1}\left(x_{s}^{n+1}, s\right)-f_{n+1}\left(x_{s}^{n}, s\right)\right|^{2} \mathrm{~d} s \\
& +\frac{6}{1-\kappa} E \int_{t_{0}}^{t}\left|g_{n+1}\left(x_{s}^{n+1}, s\right)-g_{n+1}\left(x_{s}^{n}, s\right)\right|^{2} \mathrm{~d} s \\
& +\frac{6}{1-\kappa}\left(2 \lambda+2 \lambda^{2}\left(t-t_{0}\right)\right) E \int_{t_{0}}^{t}\left|h_{n+1}\left(x_{s}^{n+1}, s\right)-h_{n+1}\left(x_{s}^{n}, s\right)\right|^{2} \mathrm{~d} s, \\
= & \kappa E \sup _{0}\left|x^{n+\tau}(s)-x^{n}(s)\right|^{2}+\frac{6}{1-\kappa}\left(t-t_{0}\right) E \int_{t_{0}}^{t}\left|f\left(x_{s}^{n+1}, s\right)-f\left(x_{s}^{n}, s\right)\right|^{2} \mathrm{~d} s \\
& +\frac{6}{1-\kappa} E \int_{t_{0}}^{t}\left|g\left(x_{s}^{n+1}, s\right)-g\left(x_{s}^{n}, s\right)\right|^{2} \mathrm{~d} s \\
& +\frac{6}{1-\kappa}\left(2 \lambda+2 \lambda^{2}\left(t-t_{0}\right)\right) E \int_{t_{0}}^{t}\left|h\left(x_{s}^{n+1}, s\right)-h\left(x_{s}^{n}, s\right)\right|^{2} \mathrm{~d} s .
\end{aligned}
$$

Noting the fact that $x^{n+1}(s)=x^{n}(s)=\xi$, for $s \in\left[t_{0}-\tau, t_{0}\right]$, we get

$$
\begin{aligned}
E \sup _{t_{0} \leq s \leq t} \mid & x^{n+1}(s)-\left.x^{n}(s)\right|^{2} \\
& \leq \frac{6\left[\left(t-t_{0}\right)+1+2 \lambda+2 \lambda^{2}\left(t-t_{0}\right)\right]}{(1-\kappa)^{2}} E \int_{t_{0}}^{t} K_{n}\left\|x_{s}^{n+1}-x_{s}^{n}\right\|^{2} \mathrm{~d} s \\
& \leq \frac{6\left[\left(t-t_{0}\right)+1+2 \lambda+2 \lambda^{2}\left(t-t_{0}\right)\right]}{(1-\kappa)^{2}} K_{n} E \int_{t_{0}}^{t} \sup _{t_{0} \leq s \leq t}\left|x^{n+1}(s)-x^{n}(s)\right|^{2} \mathrm{~d} s .
\end{aligned}
$$

From the Gronwall inequality one sees that

$$
E \sup _{t_{0} \leq s \leq t}\left|x^{n+1}(s)-x^{n}(s)\right|^{2}, \quad t_{0}<t<\tau_{n} .
$$


This means that, for $t_{0}<t<\tau_{n}$, we always have

$$
x^{n}(t)=x^{n+1}(t) .
$$

It is then deduced that $\tau_{n}$ is increasing, that is as $n \rightarrow \infty, \tau_{n} \uparrow T$ a.s. By the linear growth condition, for all almost all $\omega \in \Omega$, there exists an integer $n_{0}=n_{0}(\omega)$ such that $\tau_{n}=T$ as $n>n_{0}$. Now define $x(t)$ by $x(t)=x_{n_{0}}(t), t \in\left[t_{0}, T\right]$. Next to verify that $x(t)$ is the solution to (2.1), by (3.74) $x\left(t \wedge \tau_{n}\right)=x_{n}\left(t \wedge \tau_{n}\right)$, it follows that

$$
\begin{aligned}
x\left(t \wedge \tau_{n}\right)-G\left(x_{t \wedge \tau_{n}}^{n}\right)= & \xi(0)-G(\xi)+\int_{t_{0}}^{t \wedge \tau_{n}} f_{n}\left(x_{s}, s\right) \mathrm{d} s \\
& +\int_{t_{0}}^{t \wedge \tau_{n}} g_{n}\left(x_{s}, s\right) \mathrm{d} W(s)+\int_{t_{0}}^{t \wedge \tau_{n}} h_{n}\left(x_{s}, s\right) \mathrm{d} N(s),
\end{aligned}
$$

that is,

$$
\begin{aligned}
x\left(t \wedge \tau_{n}\right)-G\left(x_{t \wedge \tau_{n}}^{n}\right)= & \xi(0)-G(\xi)+\int_{t_{0}}^{t \wedge \tau_{n}} f\left(x_{s}, s\right) \mathrm{d} s \\
& +\int_{t_{0}}^{t \wedge \tau_{n}} g\left(x_{s}, s\right) \mathrm{d} W(s)+\int_{t_{0}}^{t \wedge \tau_{n}} h\left(x_{s}, s\right) \mathrm{d} N(s) .
\end{aligned}
$$

Letting $n \rightarrow \infty$

$$
\begin{aligned}
x(t \wedge T)-G\left(x_{t}^{n} \wedge T\right)= & \xi(0)-G(\xi)+\int_{t_{0}}^{t \wedge T} f\left(x_{s}, s\right) \mathrm{d} s \\
& +\int_{t_{0}}^{t \wedge T} g\left(x_{s}, s\right) \mathrm{d} W(s)+\int_{t_{0}}^{t \wedge T} h\left(x_{s}, s\right) \mathrm{d} N(s),
\end{aligned}
$$

that is

$$
\begin{aligned}
x(t)-G\left(x_{t}\right)= & \xi(0)-G(\xi)+\int_{t_{0}}^{t} f\left(x_{s}, s\right) \mathrm{d} s \\
& +\int_{t_{0}}^{t} g\left(x_{s}, s\right) \mathrm{d} W(s)+\int_{t_{0}}^{t} h\left(x_{s}, s\right) \mathrm{d} N(s) .
\end{aligned}
$$

We can see that $x(t)$ is the solution to $(2.1)$ and $x(t) \in \mathcal{M}^{2}\left(\left[t_{0}-\tau, T\right] ; R^{n}\right)$. The proof of existence is complete. By stopping our process, uniqueness is obtained. This completes the proof.

\section{Acknowledgments}

This research was supported with funds provided by the National Natural Science Foundation of China (no. 11102132 and no. 10732020). The authors thank two anonymous reviewers 
for their very valuable comments and helpful suggestions which improve this paper significantly.

\section{References}

[1] X. Mao, "Razumikhin-type theorems on exponential stability of neutral stochastic functionaldifferential equations," SIAM Journal on Mathematical Analysis, vol. 28, no. 2, pp. 389-401, 1997.

[2] V. Kolmanovskii, N. Koroleva, T. Maizenberg, X. Mao, and A. Matasov, "Neutral stochastic differential delay equations with Markovian switching," Stochastic Analysis and Applications, vol. 21, no. 4, pp. 819-847, 2003.

[3] X. Mao, "Razumikhin-type theorems on exponential stability of neutral stochastic functionaldifferential equations," SIAM Journal on Mathematical Analysis, vol. 28, no. 2, pp. 389-401, 1997.

[4] X. Mao, Y. Shen, and C. Yuan, "Almost surely asymptotic stability of neutral stochastic differential delay equations with Markovian switching," Stochastic Processes and their Applications, vol. 118, no. 8, pp. 1385-1406, 2008.

[5] X. Mao, "Exponential stability in mean square of neutral stochastic differential-functional equations," Systems E Control Letters, vol. 26, no. 4, pp. 245-251, 1995.

[6] X. R. Mao, Stochastic Differential Equations and Their Applications, Horwood, Chichester, UK, 1997.

[7] Q. Luo, X. Mao, and Y. Shen, "New criteria on exponential stability of neutral stochastic differential delay equations," Systems \& Control Letters, vol. 55, no. 10, pp. 826-834, 2006.

[8] S. Zhou and S. Hu, "Razumikhin-type theorems of neutral stochastic functional differential equations," Acta Mathematica Scientia. Series B, vol. 29, no. 1, pp. 181-190, 2009.

[9] J. Luo, "Fixed points and stability of neutral stochastic delay differential equations," Journal of Mathematical Analysis and Applications, vol. 334, no. 1, pp. 431-440, 2007.

[10] L.S. Wang and H. Xue, “Convergence of numerical solutions to stochastic differential delay equations with Poisson jump and Markovian switching," Applied Mathematics and Computation, vol. 188, no. 2, pp. 1161-1172, 2007.

[11] L. S. Wang, C. Mei, and H. Xue, "The semi-implicit Euler method for stochastic differential delay equations with jumps," Applied Mathematics and Computation, vol. 192, no. 2, pp. 567-578, 2007.

[12] L. Ronghua, M. Hongbing, and D. Yonghong, "Convergence of numerical solutions to stochastic delay differential equations with jumps," Applied Mathematics and Computation, vol. 172, no. 1, pp. 584-602, 2006.

[13] L. Ronghua and C. Zhaoguang, "Convergence of numerical solution to stochastic delay differential equation with Poisson jump and Markovian switching," Applied Mathematics and Computation, vol. 184, no. 2, pp. 451-463, 2007.

[14] J. W. Luo, “Comparison principle and stability of Ito stochastic differential delay equations with Poisson jump and Markovian switching," Nonlinear Analysis. Theory, Methods \& Applications. An International Multidisciplinary Journal. Series A: Theory and Methods, vol. 64, no. 2, pp. 253-262, 2006.

[15] J. Tan and H. Wang, "Mean-square stability of the Euler-Maruyama method for stochastic differential delay equations with jumps," International Journal of Computer Mathematics, vol. 88, no. 2, pp. 421-429, 2011.

[16] D. Z. Liu, G. Yang, and W. Zhang, "The stability of neutral stochastic delay differential equations with Poisson jumps by fixed points," Journal of Computational and Applied Mathematics, vol. 235, no. 10, pp. 3115-3120, 2011.

[17] J. Luo and T. Taniguchi, "The existence and uniqueness for non-Lipschitz stochastic neutral delay evolution equations driven by Poisson jumps," Stochastics and Dynamics, vol. 9, no. 1, pp. 135-152, 2009. 


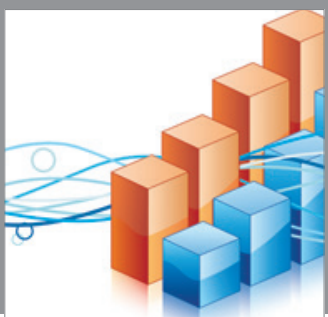

Advances in

Operations Research

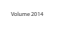

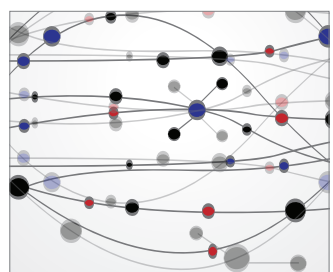

\section{The Scientific} World Journal
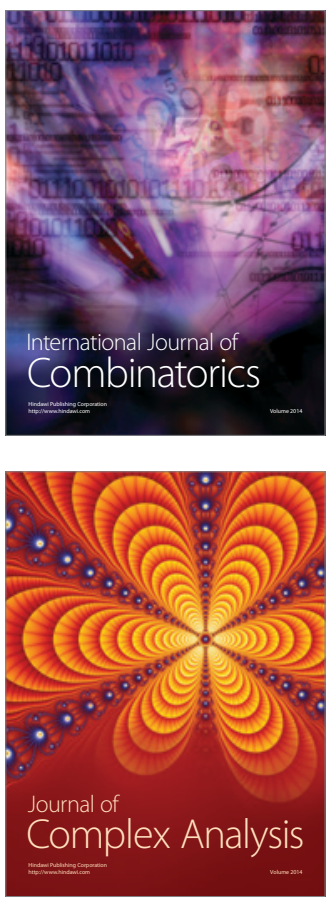

International Journal of

Mathematics and

Mathematical

Sciences
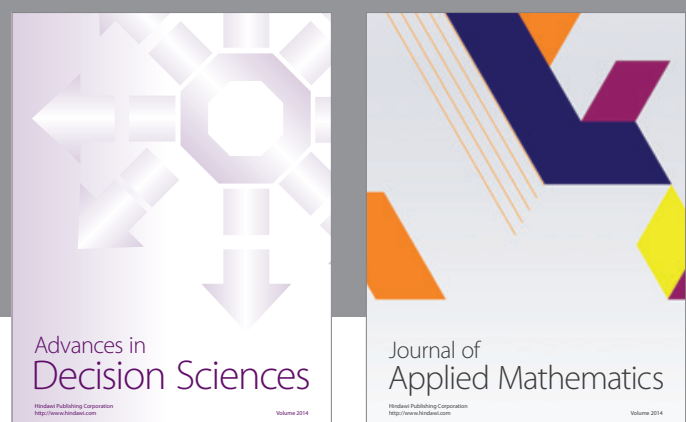

Journal of

Applied Mathematics
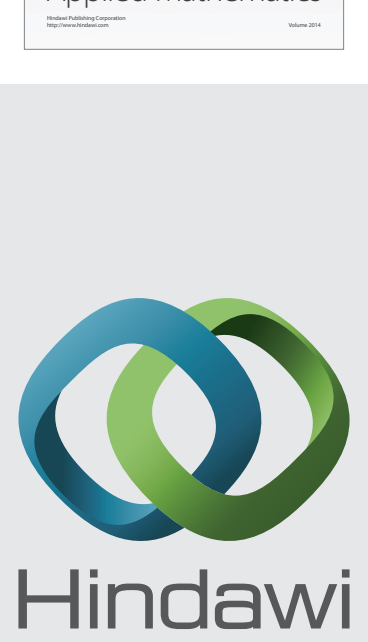

Submit your manuscripts at http://www.hindawi.com
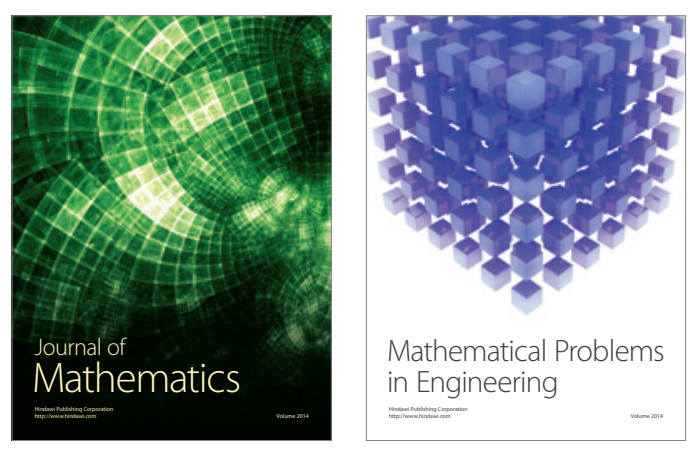

Mathematical Problems in Engineering
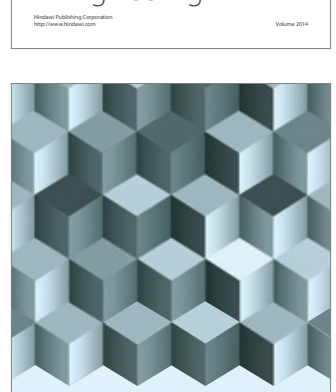

Journal of

Function Spaces
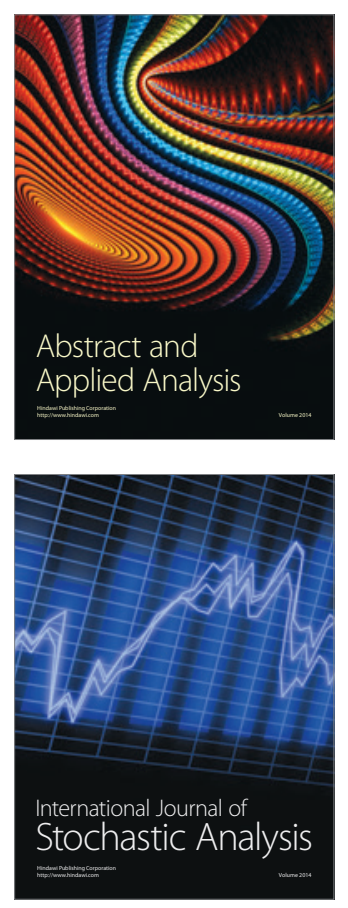

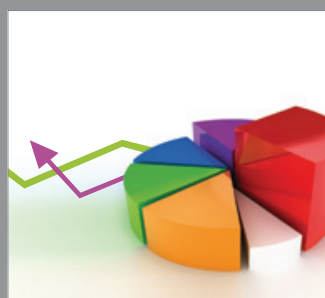

ournal of

Probability and Statistics

Promensencen
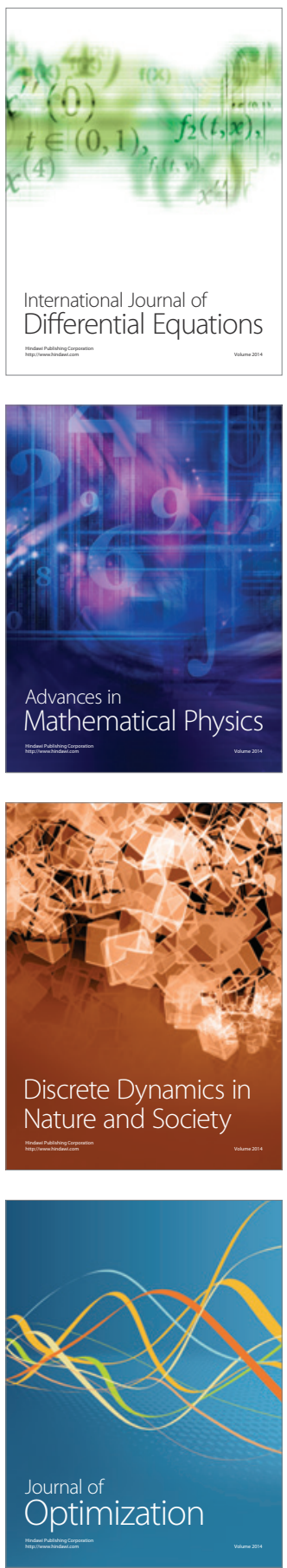